assessment and daily. Assessment of colic is often missed and prompt commencement of anti-colic therapy would benefit patient care. Standardisation of anti-emetic choice in $\mathrm{MBO}$ would be beneficial, leading to reduced inappropriate prescribing.

\section{P-135 AMENDED MOUTH CARE MATTERS PROJECT}

Jamie Yeomans. St Richard's Hospice, Worcester, UK

10.1136/spcare-2021-Hospice. 152

Background A common problem in palliative care is that of a dry and sore oral environment often resulting in infections, bad breath (halitosis), and changes in taste, therefore high quality mouth care is a very important aspect of palliative care in all care settings. When these problems are not managed effectively, they can negatively affect a patient's selfesteem, ability to communicate and socialise plus the ability to enjoy food and drinks (resulting in inadequate nutrition/hydration). It is vital therefore that mouth problems should be assessed regularly and treated as soon as possible (Marie Curie. Mouthcare, see: mariecurie.org.uk/professionals/palliative-care-knowledge-zone/symptom-control/mouth-care/).

Aims The Mouth Care Matters programme (Health Education England, see: https://mouthcarematters.hee.nhs.uk/) was designed to help deliver better clinical outcomes by evidencing the importance of good mouth care and how this positively impacts on general health and quality of life.

Methods We piloted the original '(Mouthcare Matters) Assessment and Recording Form' in the inpatient unit with the aim of providing a thorough method of assessing and addressing oral challenges experienced by our palliative/end-of-life patients (Venkatasalu, Murang, Ramasamy, et al., 2020). We then audited outcomes by publishing a scored staff feedback form to colleagues to measure effectiveness of the intervention and explore whether amendments to the tool could be implemented to make it more bespoke to our care setting (National Institute for Health and Care Excellence: Palliative care: oral. See: cks.nice.org.uk/topics/palliative-care-oral).

Results When feedback was submitted, qualitative evidence identified the need to amend the standard to truly fit the hospice model of care.

Conclusions We amended the template in the following ways:

- The addition of 'saliva' as an identifiable problem (Paine \& Snider, 2020).

- Changed 'weekly' assessment to 'daily' to reflect the pace of change some of our patients experience.

- The original tool identified low, medium and high risks but there was no advice to prompt intervention so we added an 'action checklist' section to help direct care.

We launched this amended version in April 2021 and are due to audit results in September 2021.

\section{P-136 FOUR STAGE LOW FIBRE DIETARY GUIDANCE FOR PATIENTS SUFFERING SUBACUTE MALIGNANT BOWEL OBSTRUCTION}

Sarah Onions, Nicola Wilderspin. St Richard's Hospice, Worcester, UK

10.1136/spcare-2021-Hospice.153
Background Patients with subacute malignant bowel obstruction (MBO) not amenable to surgical or interventional procedures are often highly symptomatic of pain, bloating, nausea and vomiting. Diet often exacerbates symptoms so intake is limited. UK medical treatment of $\mathrm{MBO}$ includes medicines and interventions to improve bowel transit and to manage symptoms (Ahmad, Jeffries, Longford, et al., 2015; Scottish Palliative Care Guidelines, 2020) but often little specific advice is given on low fibre diet compared to approaches worldwide (Lee, Jivraj, Wang, et al., 2019; British Columbia Cancer Agency, 2019).

Aim To develop a low fibre staged diet plan for patients with subacute or resolving $\mathrm{MBO}$. To offer patients choice and taste variety within the limitations of a clear fluid or low residue diet in order to improve symptom control, chance of resolution and quality of life.

Methods A review of literature was undertaken to better understand diet in MBO. A UK hospital's oncology dietician team use a phased model on which, with permissions, our diet is based. A collaborative approach was taken including input from the local trust dieticians and hospice multi-disciplinary team including catering and hospitality.

Results A four staged diet plan was created for our inpatient unit. Stage one, clear fluids; Stage two, liquid low fibre; Stage three, soft low fibre; Stage four, normal texture low fibre. MBO patients often start with bowel rest and move up or down the stages as tolerated. Stage four may also be used prophylactically for those at risk of developing MBO symptoms. Conclusion Most patients have welcomed specific guidance on diet. The menu provides variety despite the restrictions and many patients report significant relief of their symptoms. Patients may choose to eat off plan, as part of our 'Eat for Comfort' policy. A community $\mathrm{MBO}$ diet advice booklet has subsequently been created for those living with or at risk of $\mathrm{MBO}$, enabling patients to stay at home longer and be more in control of their symptoms.

\section{P-137 VIRTUAL REALITY IN PALLIATIVE CARE}

Jenny Warren, James Elliott, Clare Mills. Compton Care, Wolverhampton, West Midlands

\subsection{6/spcare-2021-Hospice.154}

Introduction COVID-19 pushed us as an organisation to stepup our ability to change and adapt. Our workforce has embraced the opportunities generated by digital transformations during the pandemic. Virtual Reality (VR) is revolutionising healthcare. We wanted to explore the use of VR as part of a non-pharmacological approach to symptom management and start to measure patient outcomes in a meaningful way while improving the patient's overall experience.

Actions Our initial proposal was that the use of the VR headset could positively impact on patient wellbeing. Our living well centre team commenced a pilot in conjunction with our inpatient unit. As new technology becomes more accessible use of these as part of the patient's overall plan of care is a realistic possibility even for small organisations.

Utilising freely available innovative apps the team supported patients in immersive experiences including guided relaxation meditation and visits to locations such as white sandy beaches, forests and mountain ranges. Initial outcomes were positive utilising a simple wellbeing score and numerical pain scoring. This has led us, in conjunction with a digital fabrication laboratory based at a local college, to look at 